\title{
Leading fermionic three-loop corrections to electroweak precision observables
}

\author{
Lisong Chen and Ayres Freitas \\ Pittsburgh Particle-physics Astro-physics $\&$ Cosmology Center (PITT-PACC), \\ Department of Physics 65 Astronomy, University of Pittsburgh, \\ Pittsburgh, PA 15260, U.S.A. \\ E-mail: lic114@pitt.edu, afreitas@pitt.edu
}

ABstraCt: Future electron-position colliders, such as the CEPC, FCC-ee, and ILC have the capability to dramatically improve the experimental precision for W and Z-boson masses and couplings. This would enable indirect probes of physics beyond the Standard Model at multi-TeV scales. For this purpose, one must complement the experimental measurements with equally precise calculations for the theoretical predictions of these quantities within the Standard Model, including three-loop electroweak corrections. This article reports on the calculation of a subset of these corrections, stemming from diagrams with three closed fermion loops to the following quantities: the prediction of the W-boson mass from the Fermi constant, the effective weak mixing angle, and partial and total widths of the $\mathrm{Z}$ boson. The numerical size of these corrections is relatively modest, but non-negligible compared to the precision targets of future colliders. In passing, an error is identified in previous results for the two-loop corrections to the $\mathrm{Z}$ width, with a small yet non-zero numerical impact.

Keywords: Quark Masses and SM Parameters, Scattering Amplitudes

ARXIV EPRINT: 2002.05845 


\section{Contents}

1 Introduction 1

2 Renormalization $\quad 2$

3 Definition of the observables $\quad 6$

3.1 Fermi constant $\boldsymbol{G}_{\boldsymbol{\mu}} \quad 6$

$\begin{array}{lll}3.2 & \text { Effective weak mixing angle } \sin ^{2} \theta_{\text {eff }}^{f} & 6\end{array}$

$\begin{array}{lll}3.3 & \text { Partial width } \Gamma[Z \rightarrow f \bar{f}] & 7\end{array}$

3.4 Technical aspects of the calculation 8

4 Numerical results $\quad 9$

5 Conclusions 11

\section{Introduction}

Precision measurements of processes mediated by $W$ and $Z$ bosons are crucial testbeds for the Standard Model (SM) and physics beyond the SM. Some of the most important of these electroweak precision observables (EWPOs) are (a) muon decay, mediated by a virtual $W$ boson, and (b) $e^{+} e^{-} \rightarrow f \bar{f}$, which is primarily mediated by an $s$-channel $Z$ boson for center-of-mass energies $\sqrt{s} \approx M_{\mathrm{Z}}$. Here $f$ denotes any SM lepton or quark, except the top quark. These processes receive sizable radiative corrections within the SM, which are currently known at the full two-loop level [1-22] and leading partial three- and four-loop results in powers of the top Yukawa coupling, $\alpha_{t}=\frac{y_{t}}{4 \pi}$, which have been calculated at order $O\left(\alpha_{t} \alpha_{s}^{2}\right)$ [23-25], $O\left(\alpha_{t}^{2} \alpha_{s}\right), O\left(\alpha_{t}^{3}\right)$ [26, 27], $O\left(\alpha_{t} \alpha_{s}^{3}\right)$ [28-30]. Including these corrections, the estimated theory uncertainties from missing higher orders are safely below the current experimental precision for these processes, see refs. [31-33] for recent reviews.

However, proposals for future high-luminosity $e^{+} e^{-}$colliders, such as the CEPC [34], FCC-ee [35], and ILC/Giga-Z [36] would dramatically improve the experimental precision for the relevant EWPOs, thus requiring significant additional higher-order corrections to meet the physics goals [37]. In this article, we report on the leading fermionic three-loop corrections to the EWPOs. Here "leading fermionic" refers to diagrams with the maximal number (i.e. three) of closed fermion loops. Generally, contributions with closed fermion loops are numerically enhanced since they are enhanced by powers of $m_{\mathrm{t}}$ and a large number of light fermion flavors. Technically, the leading fermionic corrections require only the computation of one-loop integrals, but care has to be taken in the derivation of the counterterms for the renormalization, as well as the description of $e^{+} e^{-} \rightarrow f \bar{f}$ as a Laurent expansion about the complex $Z$ pole [38-41]. 
Partial results for the leading fermionic three-loop corrections have been discussed in refs. [42, 43], but the proper treatment of the complex gauge boson pole was not addressed there.

In section 2, the renormalization procedure and relevant counterterms are discussed in more detail. Section 3 describes the calculation of the leading fermion three-loop corrections to the following quantities: (a) the Fermi constant for muon decay, which can be used to predict the $W$ mass, (b) the effective weak mixing angle $\sin ^{2} \theta_{\text {eff }}^{f}$, which describes the ratio of the vector and axial-vector couplings of the $Z f \bar{f}$ vertex, and (c) the partial widths for $Z \rightarrow f \bar{f}$. Numerical results are presented in section 4 , together with a discussion of their impact.

\section{Renormalization}

The calculations presented in this article are based on the on-shell renormalization scheme. In this scheme, the renormalized electromagnetic coupling is defined through the electronphoton vertex at zero momentum transfer, while the renormalized squared masses are defined at the real part of the propagator poles. For particles with a non-negligible decay width, such as the $W$ and $Z$ bosons, the propagator pole is complex and can be written as

$$
s_{0} \equiv \bar{M}^{2}-i \overline{M \Gamma},
$$

where $\bar{M}$ is the on-shell mass, while $\bar{\Gamma}$ is the particle's decay width. This definition of the mass and width is rigorously gauge-invariant [38-41], but it differs from the mass and width commonly used in the literature. Denoting the latter by $M$ and $\Gamma$, respectively, they are related according to

$$
\bar{M}=M / \sqrt{1+\Gamma^{2} / M^{2}}, \quad \bar{\Gamma}=\Gamma / \sqrt{1+\Gamma^{2} / M^{2}} .
$$

See e.g. refs. $[31,44]$ for a more detailed discussion.

Including radiative corrections, the massive gauge boson two-point function becomes

$$
D\left(p^{2}\right)=p^{2}-s_{0}+\Sigma\left(p^{2}\right)-\delta \bar{M}^{2},
$$

where $\Sigma(s)$ is the transverse part of the gauge boson self-energy, and $\delta M^{2}$ is the mass counterterm. To avoid notational clutter, we do not include a field or wavefunction renormalization for the gauge boson. Since unstable particles can only appear as internal particles in a physical process, any dependence on their field renormalization drops out in the computation of such process. ${ }^{1}$

In the on-shell scheme, $s_{0}$ is required to be a pole of the propagator, $D\left(s_{0}\right)=0$. This leads to the conditions

$$
\begin{aligned}
\delta \bar{M}^{2} & =\operatorname{Re} \Sigma\left(\bar{M}^{2}-i \overline{M \Gamma}\right), \\
\bar{\Gamma} & =\frac{1}{\bar{M}} \operatorname{Im} \Sigma\left(\bar{M}^{2}-i \overline{M \Gamma}\right) .
\end{aligned}
$$

\footnotetext{
${ }^{1}$ In our calculation, we have checked explicitly that any field renormalization counterterms cancel.
} 
By recursively inserting eq. (2.5) into (2.4) and expanding in orders of perturbation theory, the $W$-mass counterterm is given by

$$
\begin{aligned}
\delta \bar{M}_{\mathrm{W}(1)}^{2}= & \operatorname{Re} \Sigma_{\mathrm{W}(1)}\left(\bar{M}_{\mathrm{W}}^{2}\right) \\
\delta \bar{M}_{\mathrm{W}(2)}^{2}= & \operatorname{Re} \Sigma_{\mathrm{W}(2)}\left(\bar{M}_{\mathrm{W}}^{2}\right)+\left[\operatorname{Im} \Sigma_{\mathrm{W}(1)}\left(\bar{M}_{\mathrm{W}}^{2}\right)\right]\left[\operatorname{Im} \Sigma_{\mathrm{W}(1)}^{\prime}\left(\bar{M}_{\mathrm{W}}^{2}\right)\right] \\
\delta \bar{M}_{\mathrm{W}(3)}^{2}= & \operatorname{Re} \Sigma_{\mathrm{W}(3)}\left(\bar{M}_{\mathrm{W}}^{2}\right)+\left[\operatorname{Im} \Sigma_{\mathrm{W}(2)}\left(\bar{M}_{\mathrm{W}}^{2}\right)\right]\left[\operatorname{Im} \Sigma_{\mathrm{W}(1)}^{\prime}\left(\bar{M}_{\mathrm{W}}^{2}\right)\right] \\
& +\left[\operatorname{Im} \Sigma_{\mathrm{W}(1)}\left(\bar{M}_{\mathrm{W}}^{2}\right)\right]\left\{\operatorname{Im} \Sigma_{\mathrm{W}(2)}^{\prime}\left(\bar{M}_{\mathrm{W}}^{2}\right)-\left[\operatorname{Im} \Sigma_{\mathrm{W}(1)}^{\prime}\left(\bar{M}_{\mathrm{W}}^{2}\right)\right]\left[\operatorname{Re} \Sigma_{\mathrm{W}(1)}^{\prime}\left(\bar{M}_{\mathrm{W}}^{2}\right)\right]\right. \\
& -\frac{1}{2}\left[\operatorname{Im} \Sigma_{\mathrm{W}(1)}\left(\bar{M}_{\mathrm{W})}^{2}\right)\left[\operatorname{Re} \Sigma_{\mathrm{W}(1)}^{\prime \prime}\left(\bar{M}_{\mathrm{W}}^{2}\right)\right]\right\}
\end{aligned}
$$

Here and in the following the numbers in brackets denote the loop order.

For the $Z$-mass counterterm, one needs to include $\gamma-Z$ mixing effects. The $Z$ and photon fields get renormalized according to

$$
\begin{aligned}
& Z_{\mu} \rightarrow \sqrt{Z^{\mathrm{ZZ}}} Z_{\mu}+\frac{1}{2} \delta Z^{\mathrm{Z} \gamma} A_{\mu}, \\
& A_{\mu} \rightarrow \frac{1}{2} \delta Z^{\gamma \mathrm{Z}} Z_{\mu}+\sqrt{Z^{\gamma \gamma}} A_{\mu} .
\end{aligned}
$$

As already mentioned above, in the following we will simply set $Z^{\mathrm{ZZ}}, Z^{\gamma \gamma} \rightarrow 1$ for the $Z$ and photon field renormalization constants, since these drop out anyways for the physical processes discussed in this work. However, the mixing counterterms generate extra terms compared to eqs. (2.4) and (2.5):

$$
\begin{aligned}
\delta \bar{M}_{\mathrm{Z}}^{2} & =\operatorname{Re} \Sigma_{\mathrm{Z}}\left(\bar{M}_{\mathrm{Z}}^{2}-i \bar{M}_{\mathrm{Z}} \bar{\Gamma}_{\mathrm{Z}}\right)+\frac{1}{4} \bar{M}_{\mathrm{Z}}^{2}\left(\delta Z^{\gamma \mathrm{Z}}\right)^{2}, \\
\bar{\Gamma}_{\mathrm{Z}} & =\frac{1}{\bar{M}_{\mathrm{Z}}\left[1+\frac{1}{4}\left(\delta Z^{\gamma \mathrm{Z}}\right)^{2}\right]} \operatorname{Im} \Sigma_{\mathrm{Z}}\left(\bar{M}_{\mathrm{Z}}^{2}-i \bar{M}_{\mathrm{Z}} \bar{\Gamma}_{\mathrm{Z}}\right),
\end{aligned}
$$

where

$$
\begin{aligned}
& \Sigma_{\mathrm{Z}}\left(p^{2}\right)=\Sigma_{\mathrm{ZZ}}\left(p^{2}\right)-\frac{\left[\hat{\Sigma}_{\gamma \mathrm{Z}}\left(p^{2}\right)\right]^{2}}{p^{2}+\hat{\Sigma}_{\gamma \gamma}\left(p^{2}\right)} \\
& \hat{\Sigma}_{\gamma \mathrm{Z}}\left(p^{2}\right)=\Sigma_{\gamma \mathrm{Z}}\left(p^{2}\right)+\frac{1}{2} \delta Z^{\mathrm{Z} \gamma}\left(p^{2}-\bar{M}_{\mathrm{Z}}^{2}-\delta \bar{M}_{\mathrm{Z}}^{2}\right)+\frac{1}{2} \delta Z^{\gamma \mathrm{Z}} p^{2}, \\
& \hat{\Sigma}_{\gamma \gamma}\left(p^{2}\right)=\Sigma_{\gamma \gamma}\left(p^{2}\right)+\frac{1}{4}\left(\delta Z^{\mathrm{Z} \gamma}\right)^{2}\left(p^{2}-\bar{M}_{\mathrm{Z}}^{2}-\delta \bar{M}_{\mathrm{Z}}^{2}\right) .
\end{aligned}
$$

Here $\Sigma_{V_{1} V_{2}}$ is the self-energy with an incoming gauge boson $V_{1}$ and outgoing gauge boson $V_{2}$. The mixing counterterms are fixed through the conditions

$$
\hat{\Sigma}_{\gamma \mathrm{Z}}(0)=0, \quad \operatorname{Re} \hat{\Sigma}_{\gamma \mathrm{Z}}\left(\bar{M}_{\mathrm{Z}}^{2}-i \bar{M}_{\mathrm{Z}} \bar{\Gamma}_{\mathrm{Z}}\right)=0 .
$$




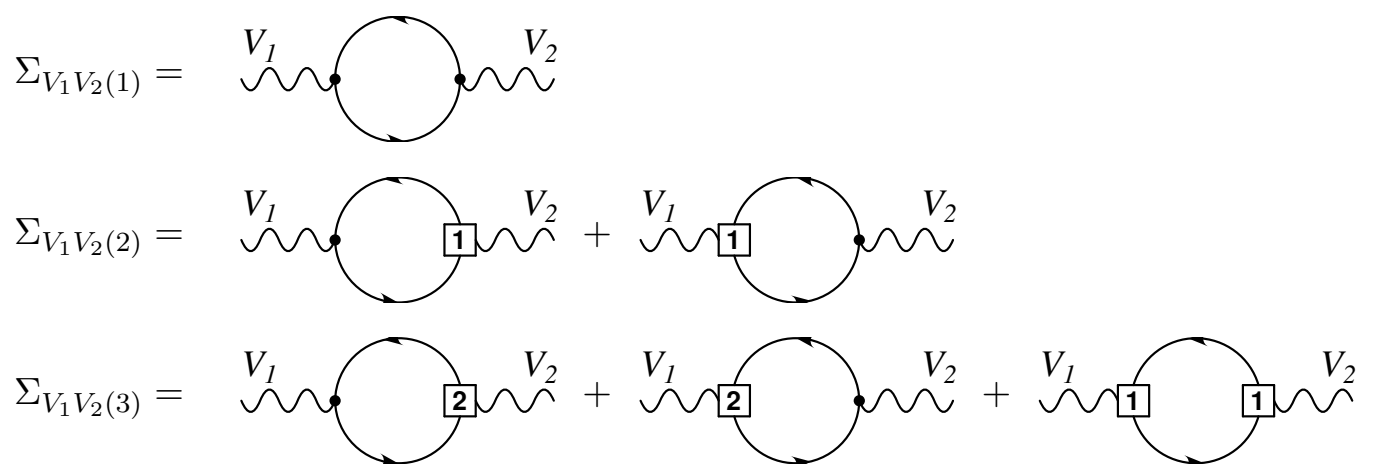

Figure 1. Diagrams with closed fermion loops contributing to self-energies at different orders. A box with number $n$ indicates a counterterm of loop order $n$. One should notice that there are no one-particle irreducible diagrams with two or three explicit closed fermion loops.

Using eqs. (2.11)-(2.16) and expanding in orders of perturbation theory yields

$$
\begin{aligned}
\delta \bar{M}_{\mathrm{Z}(1)}^{2}= & \operatorname{Re} \Sigma_{\mathrm{ZZ}(1)}\left(\bar{M}_{\mathrm{Z}}^{2}\right) \\
\delta \bar{M}_{\mathrm{Z}(2)}^{2}= & \operatorname{Re} \Sigma_{\mathrm{ZZ}(2)}\left(\bar{M}_{\mathrm{Z}}^{2}\right)+\left[\operatorname{Im} \Sigma_{\mathrm{ZZ}(1)}\left(\bar{M}_{\mathrm{Z}}^{2}\right)\right]\left[\operatorname{Im} \Sigma_{\mathrm{ZZ}(1)}^{\prime}\left(\bar{M}_{\mathrm{Z}}^{2}\right)\right] \\
& +\frac{\left[\operatorname{Im} \Sigma_{\gamma \mathrm{Z}(1)}\left(\bar{M}_{\mathrm{Z}}^{2}\right)\right]^{2}}{M_{\mathrm{Z}}^{2}}+\frac{1}{4} \bar{M}_{\mathrm{Z}}^{2}\left(\delta Z_{(1)}^{\gamma \mathrm{Z}}\right)^{2} \\
\delta \bar{M}_{\mathrm{Z}(3)}^{2}= & \operatorname{Re} \Sigma_{\mathrm{ZZ}(3)}\left(\bar{M}_{\mathrm{Z}}^{2}\right)+\left[\operatorname{Im} \Sigma_{\mathrm{ZZ}(2)}\left(\bar{M}_{\mathrm{Z}}^{2}\right)\right]\left[\operatorname{Im} \Sigma_{\mathrm{ZZ}(1)}^{\prime}\left(\bar{M}_{\mathrm{Z}}^{2}\right)\right] \\
& +\left[\operatorname{Im} \Sigma_{\mathrm{ZZ}(1)}\left(\bar{M}_{\mathrm{Z}}^{2}\right)\right]\left\{\operatorname{Im} \Sigma_{\mathrm{ZZ}(2)}^{\prime}\left(\bar{M}_{\mathrm{Z}}^{2}\right)-\left[\operatorname{Im} \Sigma_{\mathrm{ZZ}(1)}^{\prime}\left(\bar{M}_{\mathrm{Z}}^{2}\right)\right]\left[\operatorname{Re} \Sigma_{\mathrm{ZZ}(1)}^{\prime}\left(\bar{M}_{\mathrm{Z}}^{2}\right)\right]\right. \\
& -\frac{1}{2}\left[\operatorname{Im} \Sigma_{\mathrm{ZZ}(1)}\left(\bar{M}_{\mathrm{Z}}^{2}\right)\right]\left[\operatorname{Re} \Sigma_{\mathrm{ZZ}(1)}^{\prime \prime}\left(\bar{M}_{\mathrm{Z}}^{2}\right)\right] \\
& \left.-\frac{\operatorname{Im} \Sigma_{\gamma \mathrm{Z}(1)}\left(\bar{M}_{\mathrm{Z}}^{2}\right)}{\bar{M}_{\mathrm{Z}}^{2}}\left[2 \operatorname{Re} \Sigma_{\gamma \mathrm{Z}(1)}^{\prime}\left(\bar{M}_{\mathrm{Z}}^{2}\right)+\delta Z_{(1)}^{\gamma \mathrm{Z}}+\delta Z_{(1)}^{\mathrm{Z} \gamma}\right]\right\} \\
& +\frac{\operatorname{Im} \Sigma_{\gamma \mathrm{Z}(1)}\left(\bar{M}_{\mathrm{Z}}^{2}\right)}{\bar{M}_{\mathrm{Z}}^{2}}\left\{2 \operatorname{Im} \Sigma_{\gamma \mathrm{Z}(2)}\left(\bar{M}_{\mathrm{Z}}^{2}\right)-\frac{\operatorname{Im} \Sigma_{\gamma \mathrm{Z}(1)}\left(\bar{M}_{\mathrm{Z}}^{2}\right)}{\bar{M}_{\mathrm{Z}}^{2}}\left[\operatorname{Im} \Sigma_{\gamma \gamma(1)}\left(\bar{M}_{\mathrm{Z}}^{2}\right)\right]\right\} \\
& +\frac{1}{2} \bar{M}_{\mathrm{Z}}^{2} \delta Z_{(1)}^{\gamma \mathrm{Z}} \delta Z_{(2)}^{\gamma \mathrm{Z}} .
\end{aligned}
$$

The self-energies receive contributions from one-loop diagrams with counterterm insertions, see figure 1, with relevant counterterm Feynman rules shown in figure 2. It is worth noting that when inserting these into eq. (2.19), $\delta Z_{(2)}^{\gamma Z}$ drops out without needing to include an explicit expression for it.

Other relevant counterterms are given by

$$
\begin{aligned}
\delta Z_{(n)}^{\mathrm{Z} \gamma} & =0, \\
\delta Z_{e(1)} & =\frac{\alpha}{9 \pi}\left[\frac{12}{\epsilon}+\frac{50}{3}-2 L\left(m_{\mathrm{t}}^{2}\right)-10 L\left(M_{\mathrm{Z}}^{2}\right)\right]+\frac{\Delta \alpha}{2}, \\
\delta Z_{e(2)} & =\frac{3}{2}\left(\delta Z_{e(1)}\right)^{2},
\end{aligned}
$$




$$
\begin{aligned}
& \overbrace{f_{R}}^{f_{R}}=-i \gamma_{\mu} e\left(1+\delta Z_{e}\right) Q_{f}\left(1+\frac{s_{\mathrm{W}}+\delta s_{\mathrm{W}}}{2\left(c_{\mathrm{W}}+\delta c_{\mathrm{W}}\right)} \delta Z^{\mathrm{Z} \gamma}\right) \\
& \overbrace{f_{L}}^{f_{L}}=-i \gamma_{\mu} e\left(1+\delta Z_{e}\right)\left[Q_{f}\left(1+\frac{s_{\mathrm{W}}+\delta s_{\mathrm{W}}}{2\left(c_{\mathrm{W}}+\delta c_{\mathrm{W}}\right)} \delta Z^{\mathrm{Z} \gamma}\right)-\frac{I_{f}^{3}}{2\left(s_{\mathrm{W}}+\delta s_{\mathrm{W}}\right)\left(c_{\mathrm{W}}+\delta c_{\mathrm{W}}\right)} \delta Z^{\mathrm{Z} \gamma}\right] \\
& \overbrace{f_{R}}^{f_{R}}=-i \gamma_{\mu} e\left(1+\delta Z_{e}\right) Q_{f}\left(\frac{s_{\mathrm{W}}+\delta s_{\mathrm{W}}}{\left(c_{\mathrm{W}}+\delta c_{\mathrm{W}}\right)}+\frac{1}{2} \delta Z^{\gamma \mathrm{Z}}\right) \\
& \underbrace{f_{L}}_{f_{L}}=-i \gamma_{\mu} e\left(1+\delta Z_{e}\right)\left[Q_{f}\left(\frac{s_{\mathrm{W}}+\delta s_{\mathrm{W}}}{\left(c_{\mathrm{W}}+\delta c_{\mathrm{W}}\right)}+\frac{1}{2} \delta Z^{\gamma \mathrm{Z}}\right)-\frac{I_{f}^{3}}{\left(s_{\mathrm{W}}+\delta s_{\mathrm{W}}\right)\left(c_{\mathrm{W}}+\delta c_{\mathrm{W}}\right)}\right] \\
& \overbrace{f_{L}}^{f_{L}}=i \gamma_{\mu} \frac{e\left(1+\delta Z_{e}\right)}{\sqrt{2}\left(s_{\mathrm{W}}+\delta s_{\mathrm{W}}\right)}
\end{aligned}
$$

Figure 2. Feynman rules with counterterms for gauge-boson-fermion vertices. Here $I_{f}^{3}= \pm \frac{1}{2}$ for up/down-type fermions, and $Q_{f}$ is the fermion electric charge in units of $e$. $\delta Z_{e}$ and $\delta s_{\mathrm{W}}$ are the change and weak mixing angle counterterms, respectively. Furthermore, $c_{\mathrm{W}}+\delta c_{\mathrm{W}}=$ $\sqrt{1-\left(s_{\mathrm{W}}+\delta s_{\mathrm{W}}\right)^{2}}$. Note that field or wavefunction renormalization counterterms have not been included, since they are either irrelevant for the processes considered here, as explained in the text, or do not receive any contributions from closed fermion loops.

$$
\begin{aligned}
\delta Z_{e(3)} & =\frac{5}{2}\left(\delta Z_{e(1)}\right)^{3}, \\
s_{\mathrm{W}}+\delta s_{\mathrm{W}} & =\sqrt{1-\frac{\bar{M}_{\mathrm{W}}^{2}+\delta \bar{M}_{\mathrm{W}}^{2}}{\bar{M}_{\mathrm{Z}}^{2}+\delta \bar{M}_{\mathrm{Z}}^{2}}}
\end{aligned}
$$

with $L\left(m^{2}\right) \equiv \ln \frac{m^{2}}{4 \pi \mu^{2}}+\gamma_{\mathrm{E}}$. The simple results in eqs. (2.20)-(2.23) are a consequence of restricting ourselves to only closed fermion loop. The weak mixing angle counterterm is obtained by demanding that the relation $s_{\mathrm{W}}^{2}=1-\bar{M}_{\mathrm{W}}^{2} / \bar{M}_{\mathrm{Z}}^{2}$ holds to all orders. Order-byorder expressions for $\delta s_{\mathrm{W}(n)}$ can be obtained by plugging the previous expressions for the mass counterterms into (2.24), but we refrain from spelling them out here.

The symbol $\Delta \alpha$ in eq. (2.21) stems from light-fermion loop contributions in the photon vacuum polarization,

$$
\Delta \alpha=\Pi_{\mathrm{lf}}^{\gamma \gamma}\left(M_{\mathrm{Z}}^{2}\right)-\Pi_{\mathrm{lf}}^{\gamma \gamma}(0), \quad \text { where } \quad \Pi^{\gamma \gamma}\left(q^{2}\right)=\frac{\Sigma^{\gamma \gamma}\left(q^{2}\right)}{q^{2}} .
$$

$\Pi_{\mathrm{lf}}^{\gamma \gamma}\left(q^{2}\right)$ can be divided into a leptonic part, which is perturbatively calculable [46, 47], and a hadronic part that becomes non-perturbative for small $q^{2}$. Therefore the hadronic 
contribution is commonly extracted from data [48-50]. When using results from the literature for $\Delta \alpha_{\text {had }}$, one must remember that these references use $M_{Z}$ rather $\bar{M}_{\mathrm{Z}}$ in eq. (2.25). Accordingly, one must also use $M_{\mathrm{Z}}$ rather $\bar{M}_{\mathrm{Z}}$ in eq. (2.21).

\section{Definition of the observables}

\subsection{Fermi constant $G_{\mu}$}

The Fermi constant can be determined with high precision from the muon decay lifetime [51]. In the SM it is defined through

$$
G_{\mu}=\frac{\pi \alpha}{\sqrt{2} s_{\mathrm{W}}^{2} \bar{M}_{\mathrm{W}}^{2}}(1+\Delta r)
$$

where $\Delta r$ includes the contribution from radiative corrections. With $s_{\mathrm{W}}^{2}=1-\bar{M}_{\mathrm{W}}^{2} / \bar{M}_{\mathrm{Z}}^{2}$, eq. (3.1) can be used to compute a prediction for the $W$-boson mass in terms of $G_{\mu}$ and other SM parameters. Since $\Delta r$ itself depends on $\bar{M}_{\mathrm{W}}$ and $s_{\mathrm{W}}$, this is usually performed in a recursive procedure.

The corrections to $\Delta r$ from diagrams with closed fermion loops can be written as

$$
1+\Delta r=\left(\frac{1+\delta Z_{e}}{s_{\mathrm{W}}+\delta s_{\mathrm{W}}}\right)^{2} \frac{\bar{M}_{\mathrm{W}}^{2}}{\bar{M}_{\mathrm{W}}^{2}+\delta \bar{M}_{\mathrm{W}}^{2}-\Sigma_{\mathrm{W}}(0)} .
$$

It is permissible to set the momentum transfer in the $W$ propagator and in $\Sigma_{\mathrm{W}}$ to zero since $m_{\mu} \ll \bar{M}_{\mathrm{W}}$. Eq. (3.2) can be computed straightforwardly by expanding in orders of perturbation theory and using the derivations from section 2 .

\subsection{Effective weak mixing angle $\sin ^{2} \theta_{\text {eff }}^{f}$}

The effective weak mixing angle is defined in terms of the effective vector and axial-vector couplings of the $Z$-boson to an $f \bar{f}$ pair, denoted $v_{f}$ and $a_{f}$, respectively,

$$
\sin ^{2} \theta_{\text {eff }}^{f}=\frac{1}{4\left|Q_{f}\right|}\left(1+\operatorname{Re} \frac{v_{f}}{a_{f}}\right)_{s=\bar{M}_{\mathrm{Z}}^{2}} .
$$

When considering corrections with closed fermion loops, the effective couplings are obtained from the relations

$$
\begin{aligned}
& a_{f}(s)=-\frac{e\left(1+\delta Z_{e}\right) I_{3}^{f}}{2\left(s_{\mathrm{W}}+\delta s_{\mathrm{W}}\right)\left(c_{\mathrm{W}}+\delta c_{\mathrm{W}}\right)} \\
& v_{f}(s)=\frac{e\left(1+\delta Z_{e}\right)\left[I_{3}^{f}-2 Q_{f}\left(s_{\mathrm{W}}+\delta s_{\mathrm{W}}\right)^{2}\right]}{2\left(s_{\mathrm{W}}+\delta s_{\mathrm{W}}\right)\left(c_{\mathrm{W}}+\delta c_{\mathrm{W}}\right)}+e\left(1+\delta Z_{e}\right) Q_{f} \frac{\Sigma_{\gamma \mathrm{Z}}(s)-\frac{1}{2} \delta Z^{\gamma} Z_{\Sigma_{\gamma \gamma}}(s)}{s+\Sigma_{\gamma \gamma}(s)}
\end{aligned}
$$

where

$$
c_{\mathrm{W}}+\delta c_{\mathrm{W}}=\sqrt{1-\left(s_{\mathrm{W}}+\delta s_{\mathrm{W}}\right)^{2}}
$$

In writing these equations, we have used that $\delta Z^{\mathrm{Z} \gamma}=0$, see eq. (2.20), and set $Z^{\mathrm{ZZ}}, Z^{\gamma \gamma} \rightarrow 1$ as discussed above. The second term in (3.5) originates from photon- $Z$ mixing self-energies, as illustrated in figure 3 . 


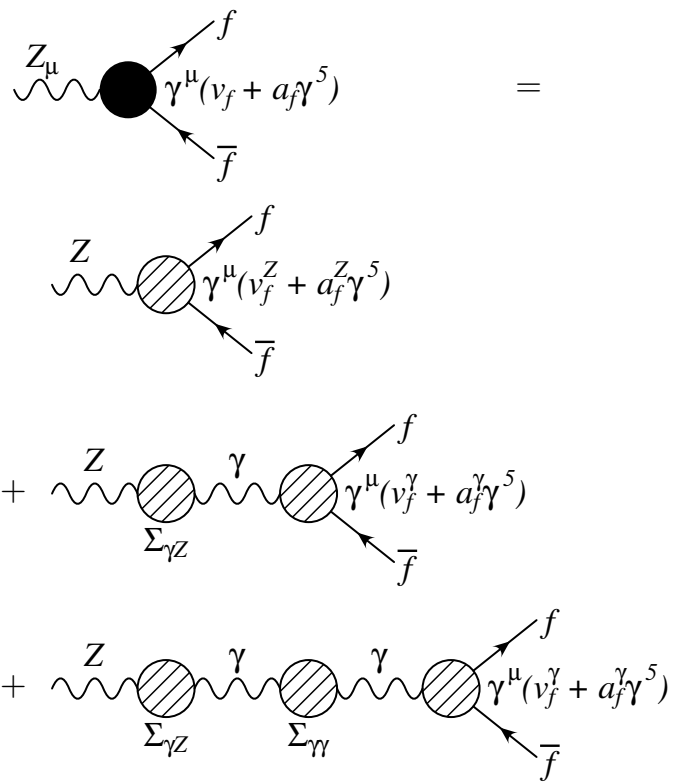

$+\ldots$
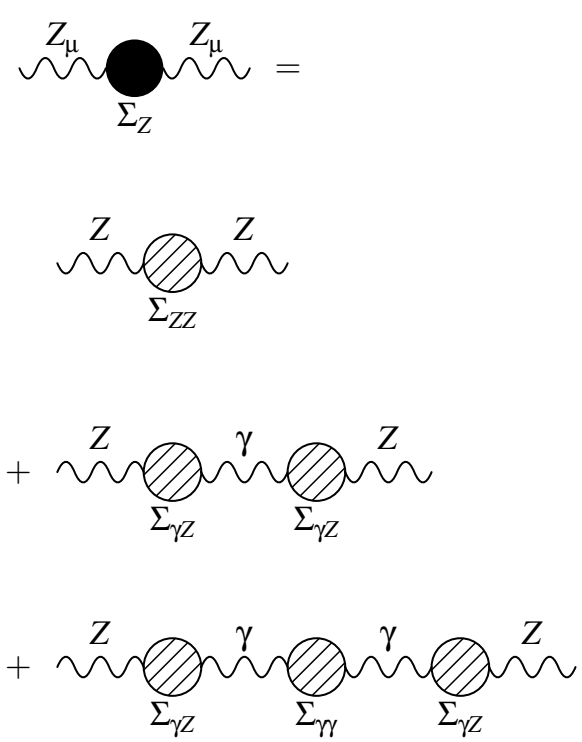

$+\ldots$

Figure 3. Decomposition of the effective $Z f \bar{f}$ vertex and $Z$ self-energy into one-particle irreducible building blocks, indicated by the hatched blobs.

\subsection{Partial width $\Gamma[Z \rightarrow f \bar{f}]$}

Recursively expanding eq. (2.12) in loop orders yields

$$
\begin{aligned}
\bar{\Gamma}_{\mathrm{Z}}= & \frac{1}{M_{\mathrm{Z}}}\left\{\operatorname{Im} \Sigma_{\mathrm{Z}(1)}+\operatorname{Im} \Sigma_{\mathrm{Z}(2)}-\left(\operatorname{Im} \Sigma_{\mathrm{Z}(1)}\right)\left(\operatorname{Re} \Sigma_{\mathrm{Z}(1)}^{\prime}\right)\right. \\
& +\operatorname{Im} \Sigma_{\mathrm{Z}(3)}-\left(\operatorname{Im} \Sigma_{\mathrm{Z}(2)}\right)\left(\operatorname{Re} \Sigma_{\mathrm{Z}(1)}^{\prime}\right) \\
& +\left(\operatorname{Im} \Sigma_{\mathrm{Z}(1)}\right)\left[\left(\operatorname{Re} \Sigma_{\mathrm{Z}(1)}^{\prime}\right)^{2}-\operatorname{Re} \Sigma_{\mathrm{Z}(2)}^{\prime}-\frac{1}{4}\left(\delta Z_{(1)}^{\gamma \mathrm{Z}}\right)^{2}-\frac{1}{2}\left(\operatorname{Im} \Sigma_{\mathrm{Z}(1)}\right)\left(\operatorname{Im} \Sigma_{\mathrm{Z}(1)}^{\prime \prime}\right)\right] \\
& +\operatorname{Im} \Sigma_{\mathrm{Z}(4)}-\left(\operatorname{Im} \Sigma_{\mathrm{Z}(3)}\right)\left(\operatorname{Re} \Sigma_{\mathrm{Z}(1)}^{\prime}\right) \\
& +\left(\operatorname{Im} \Sigma_{\mathrm{Z}(2)}\right)\left[\left(\operatorname{Re} \Sigma_{\mathrm{Z}(1)}^{\prime}\right)^{2}-\operatorname{Re} \Sigma_{\mathrm{Z}(2)}^{\prime}-\frac{1}{4}\left(\delta Z_{(1)}^{\gamma \mathrm{Z}}\right)^{2}-\left(\operatorname{Im} \Sigma_{\mathrm{Z}(1)}\right)\left(\operatorname{Im} \Sigma_{\mathrm{Z}(1)}^{\prime \prime}\right)\right] \\
& +\left(\operatorname{Im} \Sigma_{\mathrm{Z}(1)}\right)\left[-\left(\operatorname{Re} \Sigma_{\mathrm{Z}(1)}^{\prime}\right)^{3}+2\left(\operatorname{Re} \Sigma_{\mathrm{Z}(2)}^{\prime}\right)\left(\operatorname{Re} \Sigma_{\mathrm{Z}(1)}^{\prime}\right)-\operatorname{Re} \Sigma_{\mathrm{Z}(3)}^{\prime}\right. \\
& \quad-\frac{1}{2} \delta Z_{(1)}^{\gamma \mathrm{Z}} \delta Z_{(2)}^{\gamma \mathrm{Z}}+\frac{1}{2}\left(\operatorname{Re} \Sigma_{\mathrm{Z}(1)}^{\prime}\right)\left(\delta Z_{(1)}^{\gamma \mathrm{Z}}\right)^{2}-\frac{1}{2}\left(\operatorname{Im} \Sigma_{\mathrm{Z}(1)}\right)\left(\operatorname{Im} \Sigma_{\mathrm{Z}(2)}^{\prime \prime}\right) \\
& \left.\left.+\frac{3}{2}\left(\operatorname{Im} \Sigma_{\mathrm{Z}(1)}\right)\left(\operatorname{Re} \Sigma_{\mathrm{Z}(1)}^{\prime}\right)\left(\operatorname{Im} \Sigma_{\mathrm{Z}(1)}^{\prime \prime}\right)+\frac{1}{6}\left(\operatorname{Im} \Sigma_{\mathrm{Z}(1)}\right)^{2}\left(\operatorname{Re} \Sigma_{\mathrm{Z}(1)}^{\prime \prime \prime}\right)\right]\right\}_{s=\bar{M}_{\mathrm{Z}}^{2}}
\end{aligned}
$$

When neglecting light fermion masses, $\operatorname{Im} \Sigma_{Z}^{\prime \prime}=0$. Note that $\Sigma_{Z}$ includes $Z-\gamma$ mixing effects, see eq. (2.13). One can easily extend such an expansion up to higher orders.

Through the optical theorem, the imaginary part of $\Sigma_{Z}$ can be expressed in terms of the decay rate for $Z \rightarrow f \bar{f}$,

$$
\operatorname{Im} \Sigma_{\mathrm{Z}}=\frac{1}{3 \bar{M}_{\mathrm{Z}}} \sum_{f} \sum_{\text {spins }} \int d \Phi\left(\left|v_{f}\right|^{2}+\left|a_{f}\right|^{2}\right)
$$


where $a_{f}$ and $v_{f}$ are the effective axial-vector and vector couplings, defined in eqs. (3.4) and (3.5), respectively. Then one obtains the following expressions for the total and partial widths of the $\mathrm{Z}$ boson:

$$
\begin{aligned}
\bar{\Gamma}_{\mathrm{Z}}= & \sum_{f} \bar{\Gamma}_{f}, \quad \bar{\Gamma}_{f}=\frac{N_{c}^{f} \bar{M}_{\mathrm{Z}}}{12 \pi}\left[\mathcal{R}_{\mathrm{V}}^{f} F_{\mathrm{V}}^{f}+\mathcal{R}_{\mathrm{A}}^{f} F_{\mathrm{A}}^{f}\right]_{s=\bar{M}_{\mathrm{Z}}^{2}} \\
F_{\mathrm{V}}^{f}= & v_{f(0)}^{2}+2 \operatorname{Re}\left(v_{f(0)} v_{f(1)}\right)-v_{f(0)}^{2} \operatorname{Re} \Sigma_{\mathrm{Z}(1)}^{\prime} \\
+ & 2 \operatorname{Re}\left(v_{f(0)} v_{f(2)}\right)+\left|v_{f(1)}\right|^{2}-2 \operatorname{Re}\left(v_{f(0)} v_{f(1)}\right) \operatorname{Re} \Sigma_{\mathrm{Z}(1)}^{\prime} \\
+ & v_{f(0)}^{2}\left[\left(\operatorname{Re} \Sigma_{\mathrm{Z}(1)}^{\prime}\right)^{2}-\operatorname{Re} \Sigma_{\mathrm{Z}(2)}^{\prime}-\frac{1}{4}\left(\delta Z_{(1)}^{\gamma \mathrm{Z}}\right)^{2}-\frac{1}{2}\left(\operatorname{Im} \Sigma_{\mathrm{Z}(1)}\right)\left(\operatorname{Im} \Sigma_{\mathrm{Z}(1)}^{\prime \prime}\right)\right] \\
+ & 2 \operatorname{Re}\left(v_{f(0)} v_{f(3)}+v_{f(1)}^{*} v_{f(2)}\right)-\left[2 \operatorname{Re}\left(v_{f(0)} v_{f(2)}\right)+\left|v_{f(1)}\right|^{2}\right] \operatorname{Re} \Sigma_{\mathrm{Z}(1)}^{\prime} \\
+ & 2 \operatorname{Re}\left(v_{f(0)} v_{f(1)}\right)\left[\left(\operatorname{Re} \Sigma_{\mathrm{Z}(1)}^{\prime}\right)^{2}-\operatorname{Re} \Sigma_{\mathrm{Z}(2)}^{\prime}-\frac{1}{4}\left(\delta Z_{(1)}^{\gamma \mathrm{Z}}\right)^{2}-\left(\operatorname{Im} \Sigma_{\mathrm{Z}(1)}\right)\left(\operatorname{Im} \Sigma_{\mathrm{Z}(1)}^{\prime \prime}\right)\right] \\
+ & v_{f(0)}^{2}\left[-\left(\operatorname{Re} \Sigma_{\mathrm{Z}(1)}^{\prime}\right)^{3}+2\left(\operatorname{Re} \Sigma_{\mathrm{Z}(2)}^{\prime}\right)\left(\operatorname{Re} \Sigma_{\mathrm{Z}(1)}^{\prime}\right)-\operatorname{Re} \Sigma_{\mathrm{Z}(3)}^{\prime}\right. \\
& \quad-\frac{1}{2} \delta Z_{(1)}^{\gamma \mathrm{Z}} \delta Z_{(2)}^{\gamma \mathrm{Z}}+\frac{1}{2}\left(\operatorname{Re} \Sigma_{\mathrm{Z}(1)}^{\prime}\right)\left(\delta Z_{(1)}^{\gamma \mathrm{Z}}\right)^{2}-\frac{1}{2}\left(\operatorname{Im} \Sigma_{\mathrm{Z}(1)}\right)\left(\operatorname{Im} \Sigma_{\mathrm{Z}(2)}^{\prime \prime}\right) \\
& \left.+\frac{3}{2}\left(\operatorname{Im} \Sigma_{\mathrm{Z}(1)}\right)\left(\operatorname{Re} \Sigma_{\mathrm{Z}(1)}^{\prime}\right)\left(\operatorname{Im} \Sigma_{\mathrm{Z}(1)}^{\prime \prime}\right)+\frac{1}{6}\left(\operatorname{Im} \Sigma_{\mathrm{Z}(1)}\right)^{2}\left(\operatorname{Re} \Sigma_{\mathrm{Z}(1)}^{\prime \prime \prime}\right)\right]
\end{aligned}
$$

and analogously for $F_{\mathrm{A}}^{f}$. Here $N_{c}^{f}=3(1)$ for quarks (leptons), and the functions $\mathcal{R}_{\mathrm{V}, \mathrm{A}}$ are included in general to account for final-state QCD and QED corrections. When considering corrections with only closed fermion loops, $\mathcal{R}_{\mathrm{V}, \mathrm{A}}=1$.

\subsection{Technical aspects of the calculation}

Since the algebraic expressions for our results are rather lengthy, the calculation has been carried out with the help of computer algrebra tools, within the framework of MATHEMATICA. FeYnARTs 3.3 [45] has been used for the generation of diagrams and amplitudes, and FEYNCALC 9.2.0 [52] has been employed for some of the Dirac and tensor algebra. The masses and Yukawa couplings of all fermions except the top quark have been neglected. Furthermore, CKM mixing of the top quark with other quark generations has been ignored.

We have compared results for $\Delta r, \sin ^{2} \theta_{\text {eff }}^{f}$ and $\bar{\Gamma}_{f}$ with two fermionic loops with refs. $[6,7],[12]$ and $[18,19]$, respectively. Exact algebraic agreement was found, with one exception: the $\operatorname{Re} \Sigma_{Z(2)}^{\prime}$ term in the third line of (3.10), together with (2.13) and the $\gamma-Z$ mixing counterterms, leads to

$$
\operatorname{Re} \Sigma_{Z Z(2)}^{\prime}(s)-\frac{d}{d s}\left(\frac{\left[\operatorname{Im} \Sigma_{\gamma Z(1)}(s)\right]^{2}}{s}\right)
$$

The second term in eq. (3.11) was missed in refs. [18, 19]. The numerical impact of this term will be discussed in the following section. 


$$
\begin{aligned}
M_{\mathrm{Z}} & =91.1876 \mathrm{GeV} \\
\Gamma_{\mathrm{Z}} & =2.4952 \mathrm{GeV} \\
M_{\mathrm{W}} & =80.358 \mathrm{GeV} \\
\Gamma_{\mathrm{W}} & =2.089 \mathrm{GeV} \\
m_{\mathrm{t}} & =173.0 \mathrm{GeV} \\
m_{f \neq \mathrm{t}} & =0 \\
\alpha & =1 / 137.035999084 \\
\Delta \alpha & =0.05900 \\
G_{\mu} & =1.1663787 \times \bar{M}_{\mathrm{Z}}=91.1535 \mathrm{GeV} \\
&
\end{aligned}
$$

Table 1. Benchmark values for the input parameters used in the numerical analysis, based on ref. [33].

\section{Numerical results}

Let us now discuss the numerical impact of the leading fermionic three-loop corrections to the observables introduced in the previous section. For concreteness, input parameters in table 1 are used, but the results do not depend very strongly on the specific input values within experimentally allowed ranges.

With these inputs, the fermionic three-loop corrections to $\Delta r$ is found to be

$$
\Delta r_{(3)}=2.50 \times 10^{-5}
$$

This can be translated into a shift, $\Delta M_{\mathrm{W}(3)}$, of the predicted value of the $W$-boson mass in the SM, using eq. (3.1). Given that $\Delta r_{(3)}$, it is sufficient to expand eq. (3.1) up to linear order in $\Delta r_{(3)}$ and $\Delta M_{\mathrm{W}(3)}$, leading to

$$
\Delta \bar{M}_{\mathrm{W}(3)} \approx \frac{\pi \alpha \bar{M}_{\mathrm{Z}}^{2}}{2 \sqrt{2} G_{\mu} \bar{M}_{\mathrm{W}}\left(\bar{M}_{\mathrm{Z}}^{2}-2 \bar{M}_{\mathrm{W}}^{2}\right)} \Delta r_{(3)}=-0.389 \mathrm{MeV} .
$$

For the effective weak mixing angle, the fermionic three-loop correction amounts to

$$
\Delta \sin ^{2} \theta_{\mathrm{eff},(3)}^{f}=1.34 \times 10^{-5} \quad\left[M_{\mathrm{W}} \text { as indep. input }\right] .
$$

This result does not depend on the type of fermion $f$. If we assume that $M_{\mathrm{W}}$ is predicted from $G_{\mu}$, we can take into account the leading effect of the shift $\Delta M_{\mathrm{W}(3)}$ from eq. (3.1) according to

$$
\Delta^{\prime} \sin ^{2} \theta_{\mathrm{eff},(3)}^{f}=\Delta \sin ^{2} \theta_{\mathrm{eff},(3)}^{f}-\frac{\Delta \bar{M}_{\mathrm{W}(3)}^{2}}{\bar{M}_{\mathrm{Z}}^{2}}=2.09 \times 10^{-5} \quad\left[M_{\mathrm{W}} \text { from } G_{\mu}\right] .
$$

In a similar fashion, one obtains the corrections to the partial decay widths

$$
\begin{aligned}
\Delta \bar{\Gamma}_{f,(3)} & =N_{c}^{f}\left[0.105\left(I_{3}^{f}\right)^{2}-0.105 I_{3}^{f} Q_{f}+0.046 Q_{f}^{2}\right] \mathrm{MeV} \\
\Delta \bar{\Gamma}_{\ell,(3)} & =0.019 \mathrm{MeV}
\end{aligned}
$$




\begin{tabular}{|l|l|l|l|l|l|}
\hline & Current Theory & Main source & CEPC Exp & FCC-ee Exp & ILC Exp \\
\hline$M_{W}[\mathrm{MeV}]$ & 4 & $\alpha^{3}, \alpha^{2} \alpha_{s}$ & 1 & 1 & $2.5-5$ \\
$\Gamma_{Z}[\mathrm{MeV}]$ & 0.5 & $\alpha^{3}, \alpha^{2} \alpha_{s}, \alpha \alpha_{s}^{2}$ & 0.5 & 0.1 & 0.8 \\
$\sin ^{2} \theta_{\mathrm{eff}}^{l}$ & $4.3 \times 10^{-5}$ & $\alpha^{3}, \alpha^{2} \alpha_{s}$ & $2.3 \times 10^{-5}$ & $0.6 \times 10^{-5}$ & $10^{-5}$ \\
\hline
\end{tabular}

Table 2. This table demonstrates the future experimental accuracies given by CEPC, FCC-ee, and ILC with respect to the three EWPOs, along with the current theoretical uncertainties due to missing higher order [20-22, 34-37]. The methods for estimating the main sources of theory uncertainty are described in ref. [31].

$$
\begin{aligned}
& \Delta \bar{\Gamma}_{\nu,(3)}=0.026 \mathrm{MeV}, \\
& \Delta \bar{\Gamma}_{\mathrm{d},(3)}=0.041 \mathrm{MeV}, \\
& \Delta \bar{\Gamma}_{\mathrm{u},(3)}=0.035 \mathrm{MeV}, \\
& \Delta \bar{\Gamma}_{\mathrm{tot},(3)}=0.331 \mathrm{MeV}, \\
& \Delta^{\prime} \bar{\Gamma}_{f,(3)}=\Delta \bar{\Gamma}_{f,(3)}-\frac{\Delta \bar{M}_{\mathrm{W}(3)}^{2}}{\bar{M}_{\mathrm{Z}}} \times \frac{\alpha N_{c}^{f}}{6 s_{\mathrm{W}}^{4} c_{\mathrm{W}}^{4}}\left[\left(2 s_{\mathrm{W}}^{2}-1\right)\left(I_{3}^{f}\right)^{2}+2 s_{\mathrm{W}}^{4} Q_{f}\left(Q_{f}-I_{3}^{f}\right)\right] \\
& \Delta^{\prime} \bar{\Gamma}_{f,(3)}=N_{c}^{f}\left[0.090\left(I_{3}^{f}\right)^{2}-0.108 I_{3}^{f} Q_{f}+0.048 Q_{f}^{2}\right] \mathrm{MeV}, \\
& \Delta^{\prime} \bar{\Gamma}_{\ell,(3)}=0.017 \mathrm{MeV}, \\
& \Delta^{\prime} \bar{\Gamma}_{\nu,(3)}=0.022 \mathrm{MeV}, \\
& \Delta^{\prime} \bar{\Gamma}_{\mathrm{d},(3)}=0.029 \mathrm{MeV}, \\
& \Delta^{\prime} \bar{\Gamma}_{\mathrm{u},(3)}=0.024 \mathrm{MeV}, \\
& \Delta^{\prime} \bar{\Gamma}_{\text {tot },(3)}=0.255 \mathrm{MeV} .
\end{aligned}
$$

The results in eqs. (4.2), (4.5) and (4.7) are presented in terms of the gauge-invariant complex-pole definitions of the gauge-boson masses and widths. However, the corresponding corrections to the conventional (unbarred) masses and widths are the same within the precision presented above, since the translation factor in eq. (2.2) is about 1.00035, i.e. very close to 1.

When comparing the above results with the experimental determination of these quantities [33], which are dominated by measurements from LEP, SLD and LHC, one can see that the fermionic three-loop corrections are negligible compared to the experimental uncertainties. For example, the direct measurements of the $W$ mass, effective weak mixing angle, and $Z$ width are $M_{\mathrm{W}}=80.379 \pm 0.012 \mathrm{GeV}, \sin ^{2} \theta_{\mathrm{eff}}^{\ell}=0.23152 \pm 0.00016$ and $\Gamma_{\mathrm{Z} \text {,tot }}=2.4952 \pm 0.0023 \mathrm{GeV}$. These are at least one order of magnitude larger than the corrections in eqs. (4.2), (4.4) and (4.7). Our numerical results presented here are different from those in ref. [42] since we use a different renormalization scheme, but within a similar order of magnitude, both of which are expected.

However, future high-luminosity $e^{+} e^{-}$colliders, such as FCC-ee, CEPC or ILC, are anticipated to dramatically improve the experimental precision for these quantities [34-36], see table 2. It is evident that the corrections computed in this paper are important for the physics program of these machines. 
Finally, we also wish to study in the impact of the error that was found in the previous calculation of the fermionic two-loop contribution to the partial decay widths, $\Delta \bar{\Gamma}_{f,(2)}$, as discussed in section 3.4. Using the inputs from table 1, the difference amounts to

$$
\begin{aligned}
\left.\Delta \bar{\Gamma}_{f,(2)}\right|_{\text {this work }}-\left.\Delta \bar{\Gamma}_{f,(2)}\right|_{\text {Refs. [6, 7] }}= & -N_{c}^{f}\left(v_{f(0)}^{2}+a_{f(0)}^{2}\right) \bar{M}_{\mathrm{Z}} \frac{25 \alpha^{2}\left(3-8 s_{\mathrm{W}}^{2}\right)^{2}}{3888 \pi s_{\mathrm{W}}^{2} c_{\mathrm{W}}^{2}} \\
& = \begin{cases}-0.0028 \mathrm{MeV} & \text { for } f=\ell, \\
-0.0056 \mathrm{MeV} & \text { for } f=\nu, \\
-0.0126 \mathrm{MeV} & \text { for } f=d, \\
-0.0098 \mathrm{MeV} & \text { for } f=u, \\
-0.0830 \mathrm{MeV} & \text { for } f=\text { tot. }\end{cases}
\end{aligned}
$$

It turns out that the numerical impact is very small, but for the sake of consistency it is important to identify and correct this error.

\section{Conclusions}

Table 2 illustrates the comparison between the current theoretical uncertainties due to missing higher orders and the future experimental targets. For the ILC, the theoretical uncertainty for $M_{W}$ and the Z-boson width are comparable to the target precision at ILC, but the expected precision for $\sin ^{2} \theta_{\text {eff }}^{\ell}$ at ILC is smaller than the current theoretical uncertainty by roughly a factor of 4 . For the other two experiments, the experimental target precision for all observables are mostly smaller than the current theoretical uncertainties (except for the Z-boson partial width at CEPC, which is expected to have an experimental precision comparable to today's theoretical uncertainty). In particular, the target precision for the effective weak mixing angle at FCC-ee would require an improvement of the current theory error by at least one order of magnitude [37].

Hence electroweak precision measurements at future $e^{+} e^{-}$colliders, such as CEPC, FCC-ee, and ILC, require the inclusion of three-loop electroweak corrections in theoretical calculations to match the experimental precision. As a first step, this article presents results for contributions with three closed fermion loops for some of the most important electroweak precision observables (EWPOs): (i) The prediction of the $W$ mass from the muon decay rate; (ii) the ratio of vector and axial-vector $Z$-boson couplings; and (iii) $Z$-boson decay rates into different final states. Corrections with a fixed number of closed fermion loops form a UV-finite and gauge-invariant subset, and they are enhanced by powers of $m_{\mathrm{t}}$ and the large multiplicity of light fermion degrees of freedom.

Care must be taken when deriving the counterterms for the $W$ and $Z$ boson masses. Since these particles have non-negligible decay widths, their propagator poles are complex. A consistent theoretical definition of the gauge boson masses is then given by the real part of the complex propagator poles. Additional complications arise from $\gamma-Z$ mixing.

Given the large size of the final expression, the calculation has been performed with the help of computer-algebra tools. The numeric size of the leading fermionic three-loop 
corrections turns out to be relatively small for all considered EWPOs, but not negligible for the anticipated precision of CEPC, FCC-ee and ILC.

In the course of the calculation, an error was found in literature for the previously known results for the leading fermionic two-loop corrections to the $Z$-boson decay width. The numerical impact of this error is found to be very small.

Experience from electroweak two-loop calculations [6-19] shows that loop corrections with maximal number of closed fermion loops and next-to-maximal number of closed fermion loops can be of comparable size. Thus the availability of the new results in this paper, while important, does not significantly reduce the theoretical error estimates given in refs. [11, 20-22] for the relevant EWPOs. The theory uncertainties are dominated by other missing three-loop corrections which will need to be calculated to meet the goals of future $e^{+} e^{-}$colliders [37]. At the order of $\alpha^{4}$, the leading fermionic four-loop electroweak corrections can be carried out with the same approach used in this paper, but the expected size is smaller than the target precision levels in the CEPC, FCC-ee, and ILC/Giga-Z designs.

\section{Acknowledgments}

This work has been supported in part by the National Science Foundation under grant no. PHY-1820760.

Open Access. This article is distributed under the terms of the Creative Commons Attribution License (CC-BY 4.0), which permits any use, distribution and reproduction in any medium, provided the original author(s) and source are credited.

\section{References}

[1] A. Djouadi and C. Verzegnassi, Virtual very heavy top effects in LEP/SLC precision measurements, Phys. Lett. B 195 (1987) 265 [INSPIRE].

[2] A. Djouadi, $O\left(\alpha \alpha_{s}\right)$ vacuum polarization functions of the standard model gauge bosons, Nuovo Cim. A 100 (1988) 357 [inSPIRE].

[3] B.A. Kniehl, Two loop corrections to the vacuum polarizations in perturbative QCD, Nucl. Phys. B 347 (1990) 86 [INSPIRE].

[4] B.A. Kniehl and A. Sirlin, Dispersion relations for vacuum polarization functions in electroweak physics, Nucl. Phys. B 371 (1992) 141 [INSPIRE].

[5] A. Djouadi and P. Gambino, Electroweak gauge bosons selfenergies: Complete QCD corrections, Phys. Rev. D 49 (1994) 3499 [Erratum ibid. 53 (1996) 4111] [hep-ph/9309298] [INSPIRE].

[6] A. Freitas, W. Hollik, W. Walter and G. Weiglein, Complete fermionic two loop results for the $M_{W}-M_{Z}$ interdependence, Phys. Lett. B 495 (2000) 338 [Erratum ibid. 570 (2003) 265] [hep-ph/0007091] [INSPIRE].

[7] A. Freitas, W. Hollik, W. Walter and G. Weiglein, Electroweak two loop corrections to the $M_{W}-M_{Z}$ mass correlation in the standard model, Nucl. Phys. B 632 (2002) 189 [Erratum ibid. 666 (2003) 305] [hep-ph/0202131] [INSPIRE]. 
[8] M. Awramik and M. Czakon, Complete two loop bosonic contributions to the muon lifetime in the standard model, Phys. Rev. Lett. 89 (2002) 241801 [hep-ph/0208113] [INSPIRE].

[9] M. Awramik and M. Czakon, Complete two loop electroweak contributions to the muon lifetime in the standard model, Phys. Lett. B 568 (2003) 48 [hep-ph/0305248] [INSPIRE].

[10] A. Onishchenko and O. Veretin, Two loop bosonic electroweak corrections to the muon lifetime and $M_{Z}-M_{W}$ interdependence, Phys. Lett. B 551 (2003) 111 [hep-ph/0209010] [INSPIRE].

[11] M. Awramik, M. Czakon, A. Freitas and G. Weiglein, Precise prediction for the $W$ boson mass in the standard model, Phys. Rev. D 69 (2004) 053006 [hep-ph/0311148] [INSPIRE].

[12] M. Awramik, M. Czakon, A. Freitas and G. Weiglein, Complete two-loop electroweak fermionic corrections to $\sin ^{2} \theta_{\mathrm{eff}}^{\text {lept }}$ and indirect determination of the Higgs boson mass, Phys. Rev. Lett. 93 (2004) 201805 [hep-ph/0407317] [INSPIRE].

[13] M. Awramik, M. Czakon and A. Freitas, Bosonic corrections to the effective weak mixing angle at $O\left(\alpha^{2}\right)$, Phys. Lett. B 642 (2006) 563 [hep-ph/0605339] [INSPIRE].

[14] M. Awramik, M. Czakon and A. Freitas, Electroweak two-loop corrections to the effective weak mixing angle, JHEP 11 (2006) 048 [hep-ph/0608099] [INSPIRE].

[15] W. Hollik, U. Meier and S. Uccirati, The effective electroweak mixing angle $\sin ^{2} \theta_{\text {eff }}$ with two-loop fermionic contributions, Nucl. Phys. B 731 (2005) 213 [hep-ph/0507158] [INSPIRE].

[16] W. Hollik, U. Meier and S. Uccirati, The effective electroweak mixing angle $\sin ^{2} \theta_{\text {eff }}$ with two-loop bosonic contributions, Nucl. Phys. B 765 (2007) 154 [hep-ph/0610312] [INSPIRE].

[17] M. Awramik, M. Czakon, A. Freitas and B.A. Kniehl, Two-loop electroweak fermionic corrections to $\sin ^{2} \theta_{\text {eff }}^{b \bar{b}}$, Nucl. Phys. B 813 (2009) 174 [arXiv:0811.1364] [INSPIRE].

[18] A. Freitas, Two-loop fermionic electroweak corrections to the $Z$-boson width and production rate, Phys. Lett. B 730 (2014) 50 [arXiv:1310.2256] [INSPIRE].

[19] A. Freitas, Higher-order electroweak corrections to the partial widths and branching ratios of the $Z$ boson, JHEP 04 (2014) 070 [arXiv:1401.2447] [INSPIRE].

[20] I. Dubovyk, A. Freitas, J. Gluza, T. Riemann and J. Usovitsch, The two-loop electroweak bosonic corrections to $\sin ^{2} \theta_{\mathrm{eff}}^{b}$, Phys. Lett. B 762 (2016) 184 [arXiv:1607.08375] [INSPIRE].

[21] I. Dubovyk, A. Freitas, J. Gluza, T. Riemann and J. Usovitsch, Complete electroweak two-loop corrections to $Z$ boson production and decay, Phys. Lett. B $\mathbf{7 8 3}$ (2018) 86 [arXiv: 1804.10236] [INSPIRE].

[22] I. Dubovyk, A. Freitas, J. Gluza, T. Riemann and J. Usovitsch, Electroweak pseudo-observables and Z-boson form factors at two-loop accuracy, JHEP 08 (2019) 113 [arXiv: 1906.08815] [INSPIRE].

[23] L. Avdeev, J. Fleischer, S. Mikhailov and O. Tarasov, $0\left(\alpha \alpha_{s}^{2}\right)$ correction to the electroweak $\rho$ parameter, Phys. Lett. B 336 (1994) 560 [Erratum ibid. 349 (1995) 597] [hep-ph/9406363] [INSPIRE].

[24] K.G. Chetyrkin, J.H. Kühn and M. Steinhauser, Corrections of order $\mathcal{O}\left(G_{F} M_{t}^{2} \alpha_{s}^{2}\right)$ to the $\rho$ parameter, Phys. Lett. B 351 (1995) 331 [hep-ph/9502291] [INSPIRE].

[25] K.G. Chetyrkin, J.H. Kühn and M. Steinhauser, QCD corrections from top quark to relations between electroweak parameters to order $\alpha_{s}^{2}$, Phys. Rev. Lett. 75 (1995) 3394 [hep-ph/9504413] [INSPIRE]. 
[26] J.J. van der Bij, K.G. Chetyrkin, M. Faisst, G. Jikia and T. Seidensticker, Three loop leading top mass contributions to the rho parameter, Phys. Lett. B 498 (2001) 156 [hep-ph/0011373] [INSPIRE].

[27] M. Faisst, J.H. Kühn, T. Seidensticker and O. Veretin, Three loop top quark contributions to the rho parameter, Nucl. Phys. B 665 (2003) 649 [hep-ph/0302275] [inSPIRE].

[28] Y. Schröder and M. Steinhauser, Four-loop singlet contribution to the rho parameter, Phys. Lett. B 622 (2005) 124 [hep-ph/0504055] [INSPIRE].

[29] K.G. Chetyrkin, M. Faisst, J.H. Kühn, P. Maierhofer and C. Sturm, Four-Loop QCD Corrections to the Rho Parameter, Phys. Rev. Lett. 97 (2006) 102003 [hep-ph/0605201] [INSPIRE].

[30] R. Boughezal and M. Czakon, Single scale tadpoles and $O\left(G(F) m_{t}^{2} \alpha_{s}^{3}\right)$ corrections to the rho parameter, Nucl. Phys. B $\mathbf{7 5 5}$ (2006) 221 [hep-ph/0606232] [INSPIRE].

[31] A. Freitas, Numerical multi-loop integrals and applications, Prog. Part. Nucl. Phys. 90 (2016) 201 [arXiv: 1604.00406] [INSPIRE].

[32] J. Erler and M. Schott, Electroweak precision tests of the standard model after the discovery of the Higgs boson, Prog. Part. Nucl. Phys. 106 (2019) 68 [arXiv:1902.05142] [InSPIRE].

[33] Particle Data Group collaboration, Review of particle physics, Phys. Rev. D 98 (2018) 030001 [INSPIRE].

[34] CEPC Study Group collaboration, CEPC conceptual design report. Volume 2 - physics E detector, arXiv: 1811.10545 [INSPIRE].

[35] FCC collaboration, FCC-ee: the lepton collider. Future Circular Collider conceptual design report volume 2, Eur. Phys. J. ST 228 (2019) 261 [INSPIRE].

[36] A. Irles, R. Pöschl, F. Richard and H. Yamamoto, Complementarity between ILC250 and ILC-GigaZ, in Linear Collider Community Meeting, 5, 2019 [arXiv: 1905. 00220] [INSPIRE].

[37] A. Freitas et al., Theoretical uncertainties for electroweak and Higgs-boson precision measurements at FCC-ee, arXiv:1906.05379 [INSPIRE].

[38] S. Willenbrock and G. Valencia, On the definition of the $Z$ boson mass, Phys. Lett. B 259 (1991) 373 [INSPIRE].

[39] A. Sirlin, Theoretical considerations concerning the Z0 mass, Phys. Rev. Lett. 67 (1991) 2127 [INSPIRE].

[40] R.G. Stuart, Gauge invariance, analyticity and physical observables at the Z0 resonance, Phys. Lett. B 262 (1991) 113 [INSPIRE].

[41] H.G.J. Veltman, Mass and width of unstable gauge bosons, Z. Phys. C 62 (1994) 35 [INSPIRE].

[42] A. Stremplat, Zwei-Schleifen-Beiträe zu leptonischen Präzisionsobservablen, Diploma thesis, University of Karlsruhe, Karlsruhe, Germany (1998).

[43] G. Weiglein, Results for precision observables in the electroweak standard model at two loop order and beyond, Acta Phys. Polon. B 29 (1998) 2735 [hep-ph/9807222] [InSPIRE].

[44] D. Bardin, A. Leike, T. Riemann and M. Sachwitz, Energy dependent width effects in $e^{+} e^{-}$ annihilation near the $Z$ boson pole, Phys. Lett. B 206 (1988) 539 [INSPIRE]. 
[45] T. Hahn, Generating Feynman diagrams and amplitudes with FeynArts 3, Comput. Phys. Commun. 140 (2001) 418 [hep-ph/0012260] [INSPIRE].

[46] M. Steinhauser, Leptonic contribution to the effective electromagnetic coupling constant up to three loops, Phys. Lett. B 429 (1998) 158 [hep-ph/9803313] [INSPIRE].

[47] C. Sturm, Leptonic contributions to the effective electromagnetic coupling at four-loop order in QED, Nucl. Phys. B 874 (2013) 698 [arXiv: 1305.0581] [INSPIRE].

[48] F. Jegerlehner, Variations on photon vacuum polarization, EPJ Web Conf. 218 (2019) 01003 [arXiv: 1711.06089] [INSPIRE].

[49] M. Davier, A. Hoecker, B. Malaescu and Z. Zhang, A new evaluation of the hadronic vacuum polarisation contributions to the muon anomalous magnetic moment and to $\alpha\left(m_{Z}^{2}\right)$, Eur. Phys. J. C 80 (2020) 241 [Erratum ibid. 80 (2020) 410] [arXiv: 1908. 00921] [INSPIRE].

[50] A. Keshavarzi, D. Nomura and T. Teubner, $g-2$ of charged leptons, $\alpha\left(M_{Z}^{2}\right)$ and the hyperfine splitting of muonium, Phys. Rev. D 101 (2020) 014029 [arXiv:1911.00367] [INSPIRE].

[51] MuLan collaboration, Detailed report of the MuLan measurement of the positive muon lifetime and determination of the Fermi constant, Phys. Rev. D 87 (2013) 052003 [arXiv: 1211.0960] [INSPIRE].

[52] V. Shtabovenko, R. Mertig and F. Orellana, New developments in FeynCalc 9.0, Comput. Phys. Commun. 207 (2016) 432 [arXiv: 1601.01167] [INSPIRE]. 